\title{
ANALYSIS OF THE CONVERGENCE PROPERTIES OF RUBENSTEIN'S METHOD FOR THE DETERMINATION OF THE LOWER MODES OF VIBRATION OF A MULTI-DEGREE OF FREEDOM SYSTEM
}

\author{
By M. E. J. O'ḰELly
}

\begin{abstract}
This paper analyzes the convergence properties of a method, recently proposed by Rubenstein, for the determination of the lower modes of vibration of a multi-degree of freedom system from a reduced eigenvalue problem. It is shown that under certain conditions the method converges to the exact eigenvalues. It does not have global convergence and hence some care must be exercised when using it.
\end{abstract}

\section{INTRODUCTION}

In a recent paper entitled, "Determination of the first $n$ Modes of a Tall Building from a Reduced Eigenvalue Problem," Bull. Seis. Soc. Amer. 54, p. 1233, Professor Rubenstein develops a method for determining the lower modes of vibration of a large order multi-degree of freedom system by considering the exact modes of a lower order system which is derived from the higher order system. The main advantage of Rubenstein's method is the reduction, over the conventional methods, of the computer time required to calculate the modes of the system. In this paper, the question of the convergence of Rubenstein's method to a particular mode shape will be discussed, and it will be shown that the method converges but the choice of the lower order system is critical as far as convergence to any particular mode is concerned.

\section{THEORY}

The equations of motion of a multi-degree of freedom, undamped, linear dynamic passive system may be written as

$$
[M]\{\ddot{x}\}+[K]\{x\}=\{f(t)\}
$$

where $[M]$ and $[K]$ are symmetric $N \times N$ matrices, $[M]$ is positive definite and $[K]$ is at least non-negative definite, $[M]$ is the inertia matrix and $[K]$ is the stiffness matrix. $\{x\}$ is a $N \times 1$ column vector, the elements of which denote the displacement of the mass points of the system.

$\{f(t)\}$ is a $N \times 1$ column vector, the elements of which denote the external forcing functions acting at the mass points of the system.

A considerable amount of work has been done on systems of type (1) and the following results, of use later in this paper, are well known.

(i) As $[M]$ and $[K]$ are symmetric and $[M]$ is positive definite, there exists a transformation $[\Phi]$ such that

$$
\begin{array}{ll}
{[\Phi]^{T}[M][\Phi]=I} & \text { the identity matrix } \\
{[\Phi]^{T}[K][\Phi]=\left[\omega_{1}^{2}\right]} & \text { a diagonal matrix. }
\end{array}
$$


(ii) By using the $N \times N$ linear transformation [ $\Phi]$ it is possible to uncouple (1) in $N$ space.

(iii) (1) possesses classical normal $\operatorname{modes}^{(1)}$, i.e., the columns of $[\Phi]$ are such that;

(a) It is possible to express them as a complex scalar times a vector, the elements of which are real numbers. The eigenvalue $\omega_{i}$ associated with each mode is real.

(b) They form an orthonormal set of vectors with respect to $[M]$ as shown in (i) above.

(c) It is possible to excite (1) to vibrate in a pure mode by free vibration with a suitable choice of initial conditions, by a particular distribution of forcing functions with zero initial conditions or by a combination of initial conditions and forcing function.

To understand the basis of Rubenstein's method, it is essential to first appreciate the intimate relationship that exists between the Rayleigh Quotient, the RayleighRitz Method applied to the Rayleigh Quotient, and the ordinary eigenvalue-eigenfunction equation of the system.

Consider the Rayleigh Quotient associated with (1)

$$
\omega_{x}^{2}=\frac{\{x\}^{T}[K]\{x\}}{\{x\}^{T}[M]\{x\}} \quad \text { for any }\{x\}
$$

It is well known that

$$
\omega_{1}^{2} \leqq \omega_{x}^{2} \leqq \omega_{N}^{2}
$$

where $\omega_{1}$ and $\omega_{N}$ are the lowest and highest eigenvalues of (1), respectively.

To apply the Rayleigh-Ritz procedure to (2), consider $\{x\}$ to be a vector with elements $x_{i}, i=1,2, \cdots N$ and determine the variation in $\omega_{x}{ }^{2}$ as $x_{i}$ is changed infinitesimally about a stationary point of $\omega_{x}^{2}$. This corresponds to the more usual method of taking the following sum of base vectors

$$
\{x\}=\sum_{i=1}^{N} x_{i}\left\{e_{i}\right\}
$$

where the vectors $\left\{e_{i}\right\}$ have all zero elements with the exception of their $i$-th element which is 1 , and determining the best possible set of $x_{i}$ 's from variational considerations.

$$
\frac{\partial \omega_{x}^{2}}{\partial x_{i}}=\frac{2\{x\}^{T}[M]\{x\}([K]\{x\})_{i}-2\{x\}^{T}[K]\{x\}([M]\{x\})_{i}}{\left(\{x\}^{T}[M]\{x\}\right)^{2}} \quad i=1,2, \cdots N
$$

where $([K]\{x\})_{i}$ and $([M]\{x\})_{i}$ are the $i$-th elements of the vectors $[K]\{x\}$ and $[M]\{x\}$ respectively. From $(5)$

$$
\frac{\partial \omega_{x}^{2}}{\partial x_{i}}=0 \quad \text { if }
$$




$$
\{x\}^{T}[M]\{x\}([K]\{x\})_{i}=\{x\}^{T}[K]\{x\}([M]\{x\})_{i} \quad i=1,2, \cdots N
$$

Simplifying (6), it may be seen that $\{x\}$ must be such that

$$
[M]\{x\}=\frac{\{x\}^{T}[M]\{x\}}{\{x\}^{T}[K]\{x\}}[K]\{x\}
$$

which is in the form of the usual eigenvalue-eigenvector equation associated with system (1).

\section{Development of Rubenstein's Method}

It will now be shown that Rubenstein's reduced system is nothing more than the original system restricted to vibrate in such a manner that its motion is a linear combination of $n,(n<N), N \times 1$ column vectors.

$$
\text { Let }\{x\}=[Q]_{N \times n}\{y\}
$$

where $[Q]_{N \times n}$ is a $N \times n$ (rectangular) matrix and $\{y\}$ is a $n \times 1$ column vector. Substituting (8) into (1)

$$
[M][Q]_{N \times n}\{\ddot{y}\}+[K][Q]_{N \times n}\{y\}=\{f(t)\}
$$

Premultiply (9) by $\left([Q]_{N \times r}\right)^{T}$, a $n \times N$ rectangular matrix

$$
\left([Q]_{N \times n}\right)^{T}[M][Q]_{N \times n}\{\ddot{y}\}+\left([Q]_{N \times n}\right)^{T}[K][Q]_{N \times n}\{y\}=\left([Q]_{N \times n}\right)^{T}\{f(t)\}
$$

In Rubenstein's notation, let

$$
\begin{array}{ll}
\left([Q]_{N \times n}\right)^{T}[M][Q]_{N \times n}=[M]_{q} & \text { a } n \times n \text { matrix } \\
\left([Q]_{N \times n}\right)^{T}[K][Q]_{N \times n}=[K]_{q} & \text { a } n \times n \text { matrix }
\end{array}
$$

Hence the homogeneous part of (10) may be written as

$$
[M]_{q}\{\ddot{y}\}+[K]_{q}\{y\}=0
$$

Let the columns of $[\Phi]$, defined under (i) above, be designated by $\phi_{1}, \phi_{2} \cdots \phi_{N}$, i.e., the $\phi_{i}$ 's $i=1,2, \cdots N$ are eigenvectors of (1).

If the $n$ columns of $[Q]_{N \times n}$ are in fact eigenvectors of (1), then from (i) above it may be seen that the system given by (11) is completely uncoupled in $n$ space, being equal to

$$
I\{\ddot{y}\}+\left[\omega^{2}\right]\{y\}=0
$$

where the diagonal elements of the diagonal matrix [ $\left.\omega^{2}\right]$ depend on which set of $n$ of the $N$ eigenvectors of (1) are taken as columns of $[Q]_{N \times n}$. The eigenvectors of 
(12) are

$$
\left\{y_{i}\right\}=\left\{e_{i}\right\}
$$

and the eigenvalues are the diagonal elements of [ $\omega^{2} \backslash$. Hence, from (8) it may be seen that, if the columns of $[Q]_{N \times n}$ are in fact eigenvectors of the large order system specified by (1) the reduced system given by (11) reproduces the eigenvectors and eigenvalues of the large order system. Therefore Rubenstein's method does not possess global convergence to the lower modes of the system, or in other words the matrix $[Q]_{N \times n}$ plays a crucial role as to what results can be obtained from the method. In general, when dealing with numerical schemes of analysis, there are two main considerations; (i) if the scheme approximates the desired result and (ii) if it is possible to refine the approximation by successive iterations of the scheme. Below it will be shown that if the columns of $[Q]_{N \times n}$ are within order $\epsilon(\epsilon \ll 1)$ of the eigenvectors of (1), Rubenstein's method gives after one iteration approximations to the eigenvectors of (1) to order $\epsilon^{2}$. Hence, provided the initial estimates of the eigenvectors of $(1)$, which form the columns of $[Q]_{N \times n}$, are within order $\epsilon$ of their true value, the scheme proposed by Rubenstein can under fairly general conditions converge arbitrarily close after a number of iterations to exact eigenvectors of the system. The nature of the required general conditions will be seen immediately from the following analysis.

\section{Perturbation Analysis of Rubenstein's Method}

Since global convergence of the method does not exist, recourse must be had to a perturbation analysis to determine the convergence properties of the scheme. As the $\phi_{i}$ 's $i=1,2, \cdots N$ are the eigenvectors of the definite eigenvalue problem specified by (1), they form a complete set in $N$ space and hence any $\phi_{i}{ }^{*}, i=1$, $2, \cdots n$ may be expanded in the following manner

$$
\left\{\phi_{i}^{*}\right\}=\left\{\phi_{i}\right\}+\sum_{j}^{\prime} a_{i j}^{*}\left\{\phi_{j}\right\} \quad i=1,2, \cdots n
$$

where

$$
\sum_{j}^{N^{\prime}} b_{i j}=\sum_{\substack{j=1 \\ \neq i}}^{N} b_{i j}
$$

If the $\left\{\phi_{i}^{*}\right\}$ 's are "close" to the exact eigenvectors of (1), the coefficients $a_{i j}^{*}$ must be small, i.e.,

$$
\left\{\phi_{i}\right\}^{*}=\left\{\phi_{i}\right\}+\epsilon \sum_{j}^{N^{\prime}} a_{i j} \phi_{j},|\epsilon| \ll 1
$$

From (15)

$$
[M]_{q}=\left([Q]_{N \times n}\right)^{T}[M][Q]_{N \times n}
$$


where

$$
[Q]_{n \times N}=\left[\phi_{1}^{*} \phi_{2}^{*} \phi_{3}^{*} \cdots \phi_{n}^{*}\right]
$$

Using the orthonormality conditions of the $\phi_{i}{ }^{\prime}$ s, $i=1,2, \cdots N$ it may be seen that $[M]_{q}=\left[\begin{array}{c}1+\epsilon^{2} \sum_{j}^{N^{\prime}} a_{1 j}^{2} \epsilon\left(a_{12}+a_{21}\right)+\epsilon^{2} \sum_{j}^{N^{\prime \prime}} a_{1 j} a_{2 j} \cdots \epsilon\left(a_{1 n}+a_{n 1}\right)+\epsilon^{2} \sum_{j}^{N^{\prime \prime}} a_{1 j} a_{n j} \\ \epsilon\left(a_{21}+a_{12}\right)+\epsilon^{2} \sum_{j}^{N^{\prime \prime}} a_{1 j} a_{2 j} 1+\epsilon^{2} \sum_{j}^{N^{\prime}} a_{2 j} \ldots \ldots \cdots \\ \vdots \\ \vdots \\ \vdots \\ \epsilon\left(a_{n 1}+a_{1 n}\right)+\epsilon^{2} \sum_{j}^{N^{\prime \prime}} a_{n j} a_{1 j} \ldots \ldots \cdots \cdots \\ \vdots \\ \vdots \\ \vdots \\ \vdots\end{array}\right]$

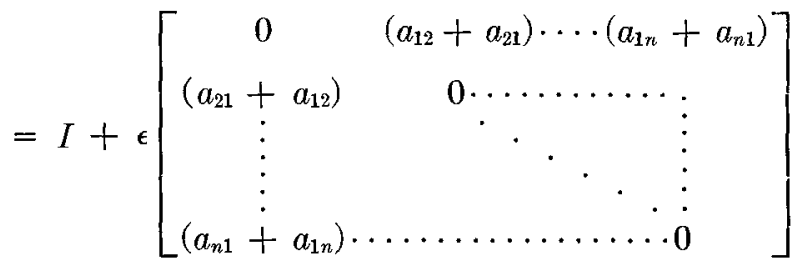

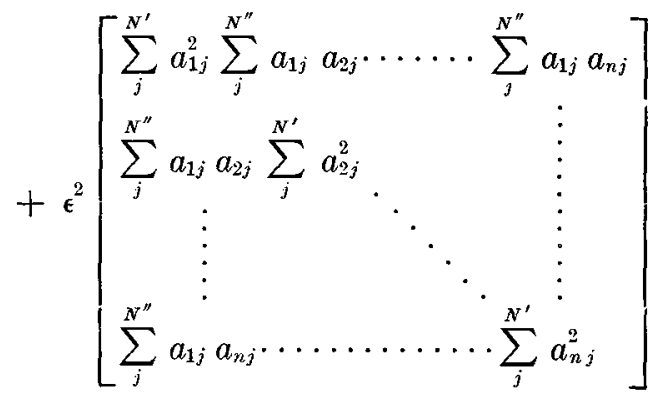

where

$$
\begin{aligned}
\sum_{j}^{N^{\prime \prime}} a_{i j} a_{l j} & =\sum_{\substack{j=1 \\
\neq i, l}}^{N} a_{i j} a_{l j} \\
& =I+\epsilon\left[A_{1}\right]+\epsilon^{2}\left[A_{2}\right]
\end{aligned}
$$

where

$$
\begin{array}{rlrl}
A_{1 i j} & =\left(a_{i j}+a_{j i}\right) & i, j=1,2, \cdots n, i \neq j \\
& =0 & i & =j
\end{array}
$$




$$
A_{2 i j}=\sum_{i}^{N^{\prime \prime}} a_{i l} a_{j l} \quad l, j=1,2, \cdots n .
$$

Similarly, it may be shown that

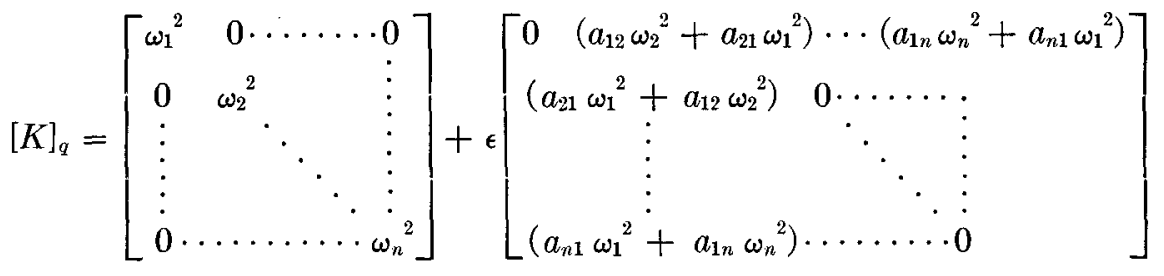

$$
\begin{aligned}
& +\epsilon^{2}\left[\begin{array}{c}
\sum_{j}^{N^{\prime}} a_{1 j}^{2} \omega_{j}^{2} \sum_{j}^{N^{\prime \prime}} a_{1 j} a_{2 j} \omega_{j}{ }^{2} \ldots \ldots \sum_{j}^{N^{\prime \prime}} a_{1 j} a_{n j} \omega_{j}{ }^{2} \\
\sum_{j}^{N^{\prime \prime}} a_{2 j} a_{1 j} \omega_{j}^{2} \sum_{j}^{N^{\prime}} a_{2 j}^{2} \omega_{j}{ }^{2} \ldots \ldots \ldots \vdots \\
\vdots \\
\vdots \\
\sum_{j}^{N^{\prime \prime}} a_{n j} a_{1 j} \omega_{j}{ }^{2} \ldots \ldots \ldots \sum_{j}^{N^{\prime}} a_{n j}^{2} \omega_{j}^{2}
\end{array}\right] \\
& {[K]_{q}=[\bar{K}]+\epsilon\left[B_{1}\right]+\epsilon^{2}\left[B_{2}\right]} \\
& \bar{K}_{i j}=0 \quad i \neq j \quad i, j=1,2, \cdots n \\
& =\omega_{i}^{2} \quad i=j \\
& B_{1 i j}=a_{i j} \omega_{j}{ }^{2}+a_{j i} \omega_{i}{ }^{2} \quad i \neq j \quad i, j=1,2, \cdots n \\
& =0 \quad i=j \\
& B_{2 i j}=\sum_{l}^{N^{\prime \prime}} a_{i l} a_{j l} \omega_{l}^{2} \\
& l, j=1,2, \cdots n
\end{aligned}
$$

The eigenvalue-eigenvector problem associated with the reduced system

$$
[M]_{q}\{\ddot{X}\}+[K]_{q}\{X\}=0
$$

may now be written as

$$
\lambda_{i}^{*}\left[I+\epsilon\left[A_{1}\right]+\epsilon^{2}\left[A_{2}\right]\right]\left\{q_{i}\right\}=\left[-[\bar{K}]-\epsilon\left[B_{1}\right]-\epsilon^{2}\left[B_{2}\right]\right]\left\{q_{i}\right\}
$$

where $\left\{q_{i}\right\}$ is a $n \times 1$ eigenvector, with associated eigenvalue $\lambda_{i}{ }^{*} i=1,2, \cdots n$. Assuming that it is possible to expand $\left\{q_{i}\right\}$ and $\lambda_{i}{ }^{*}, i=1,2, \cdots n$, in a power series in $\epsilon$, i.e.,

$$
\begin{aligned}
& \left\{q_{i}\right\}=\left\{z_{i}\right\}+\epsilon\left\{p_{i}\right\}+\epsilon^{2}\left\{\varphi_{i}\right\}+\cdots \\
& \lambda_{i}{ }^{*}=\lambda_{i}+\epsilon \theta i+\epsilon^{2} v_{i}+\cdots
\end{aligned}
$$


Equation (21) implies that $\left\{q_{i}\right\}$ and $\lambda_{i}{ }^{*}$ vary analytically with $\epsilon$ or, in a practical sense, a small change in $\epsilon$ produces only a small change in $\left\{q_{i}\right\}$ and $\lambda_{i}{ }^{*}$. This assumption may be shown to be valid in the case in which the diagonal elements of $[\bar{K}]$ are distinct. In the case in which the diagonal elements of $[\bar{K}]$ are not distinct, the vectors $\left\{z_{i}\right\}$ associated with the repeated diagonal elements are not unique in direction and a series such as (21) is not in general valid. But (20) is a positive definite eigenvalue-eigenvector problem as $[M]_{q}$ may easily be shown to be a positive definite matrix and $[K]_{q}$ is symmetric. Hence, even in the case of repeated diagonal elements of $[\bar{K}]$ (repeated eigenvalues of $[M]^{-1}[K]$, the $N \times N$ system ) there exists a full complement of ordinary eigenvectors. The difficulty is to determine the starting vector $\left\{z_{i}\right\}$ such that series $(21)$ is valid. This problem has been solved by Dr. T. K. Caughey and the present author. ${ }^{(2)}$ Here it will be assumed that the diagonal elements of $[\bar{K}]$ are distinct to simplify the algebraic manipulations.

Substituting (21) into (20) and equating the coefficients of the various powers of $\epsilon$ on both sides of the resulting equation

Zeroth order in $\epsilon$

$$
\lambda_{i}[I]\left\{z_{i}\right\}+[\bar{K}]\left\{z_{i}\right\}=0
$$

First order in $\epsilon$

$$
\lambda_{i}[I]\left\{p_{i}\right\}+[\bar{K}]\left\{p_{i}\right\}=-\lambda_{i}\left[A_{1}\right]\left\{z_{i}\right\}-\left[B_{1}\right]\left\{z_{i}\right\}-\theta_{i}[I]\left\{z_{i}\right\}
$$

Second order in $\epsilon$

$$
\begin{aligned}
\lambda_{i}[I]\left\{\varphi_{i}\right\}+[\bar{K}]\left\{\varphi_{i}\right\}=-\lambda_{i}\left[A_{1}\right]\left\{p_{i}\right\} & -\lambda_{i}\left[A_{2}\right]\left\{z_{i}\right\}-\theta_{i}[I]\left\{p_{i}\right\} \\
& -\theta_{i}\left[A_{1}\right]\left\{z_{i}\right\}-v_{i}[I]\left\{z_{i}\right\}-\left[B_{1}\right]\left\{p_{i}\right\}-\left[B_{2}\right]\left\{z_{i}\right\}
\end{aligned}
$$

From (22) as the diagonal elements of $[\bar{K}]$ are distinct

$$
\begin{aligned}
\left\{z_{i}\right\} & =\left\{e_{i}\right\} \\
\lambda_{i} & =-\omega_{i}{ }^{2} \quad i=1,2, \cdots n
\end{aligned}
$$

From (23) on substituting (25)

$$
-\omega_{i}{ }^{2}[I]\left\{p_{i}\right\}+[\bar{K}]\left\{p_{i}\right\}=\omega_{i}{ }^{2}\left\{\begin{array}{c}
a_{1 i}+a_{i 1} \\
a_{2 i}+a_{i 2} \\
\vdots \\
0(i \text { throw }) \\
\vdots \\
a_{n i}+a_{i n}
\end{array}\right\}-\left\{\begin{array}{c}
a_{1 i} \omega_{i}{ }^{2}+a_{i 1} \omega_{1}{ }^{2} \\
a_{2 i} \omega_{i}{ }^{2}+a_{i 2} \omega_{2}{ }^{2} \\
\vdots \\
0(i \text { th row }) \\
\vdots \\
a_{n i} \omega_{i}{ }^{2}+a_{i n} \omega_{n}{ }^{2}
\end{array}\right\}-\theta_{i}\left\{e_{i}\right\}
$$

From (26) it may be seen that

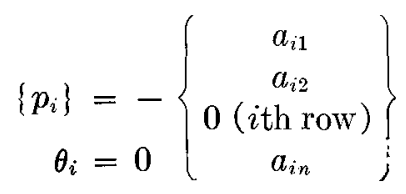


Hence, from (21), (25) and (27) $\left\{q_{i}\right\}$ and $\lambda_{i}{ }^{*} i=1,2, \cdots n$ may be written as

$$
\begin{aligned}
\left\{q_{i}\right\} & =\left\{e_{i}\right\}+\epsilon\left\{p_{i}\right\}+\epsilon^{2}\left\{\varphi_{i}\right\}+\cdots \\
\lambda_{i}^{*} & =-\omega_{i}{ }^{2}+\epsilon^{2} v_{i}+\cdots
\end{aligned} \quad i=1,2, \cdots n
$$

To relate these eigenvectors and eigenvalues to the eigenvectors and eigenvalues of the original $N \times N$ system, use is made of the transformation given by equation (8)

$$
\{x\}=[Q]_{N \times n}\{y\}
$$

On substituting (15), (16) and (28) into (29), the eigenvectors and eigenvalues of the original system are obtained

$$
\begin{aligned}
\left\{x_{i}\right\}=\left[\phi_{1}+\epsilon \sum_{j}^{N^{\prime}} a_{1 j} \phi_{j}, \phi_{2}+\epsilon \sum_{j}^{N^{\prime}} a_{2 j} \phi_{j}, \cdots \phi_{n}+\epsilon \sum_{j}^{N^{\prime}} a_{n j} \phi_{j}\right] \\
\cdot\left\{\left\{e_{i}\right\}+\epsilon\left\{p_{i}\right\}+\epsilon^{2}\left\{\varphi_{i}\right\}+\cdots\right\}=\phi_{i}+\epsilon \sum_{j}^{N^{\prime}} a_{i j} \phi_{j}-\epsilon \sum_{j}^{n^{\prime}} a_{i j} \phi_{j} \\
+\epsilon^{2}\{\quad\} \cdots=\phi_{i}+\epsilon \sum_{j=n+1}^{N} a_{i j} \phi_{j}+\epsilon^{2}\{\} \quad i=1,2, \cdots n
\end{aligned}
$$

Hence, if the vectors $\phi_{i}{ }^{*} i=1,2, \cdots, n$, are given by expressions of type (15), Rubenstein's method gives after one iteration eigenvalues and eigenvectors that are in general better approximations to the correct eigenvalues and eigenvectors than the initial set of approximations. To determine precisely the nature of the approximation after one iteration recourse must be had to (24), the equation equating to zero the coefficient of the second order times in $\epsilon$ in expansion (20).

Substituting (25) and (27) into (24)

$$
\begin{aligned}
& -\omega_{i}{ }^{2} I\left\{\varphi_{i}\right\}+\bar{K}\left\{\varphi_{i}\right\}=-\omega_{i}{ }^{2}\left\{\begin{array}{cc}
\sum_{j}^{n^{\prime \prime}} a_{i j} a_{1 j} & +\sum_{j}^{n^{\prime \prime}} a_{i j} a_{j 1} \\
\vdots & n_{j}^{n^{\prime \prime}} a_{i j}+\sum_{j} a_{i j} a_{j i} \\
\vdots & n^{\prime \prime} \\
\sum_{j}^{n^{\prime \prime}} a_{i j} a_{n j}+\sum_{j} a_{i j} a_{j n}
\end{array}\right\}+\omega_{i}{ }^{2}\left\{\begin{array}{c}
\sum_{j}^{N^{\prime \prime}} a_{1 j} a_{i j} \\
\vdots \\
\sum_{j}^{N^{\prime}} a_{i j}^{2} \\
\vdots \\
\sum_{j}^{N^{\prime \prime}} a_{n j} a_{i j}
\end{array}\right\} \\
& -\left\{\begin{array}{c}
\sum_{j}^{N^{\prime \prime}} a_{1 j} a_{i j} \omega_{j}^{2} \\
\vdots \\
\sum_{j}^{N^{\prime}} a_{i j}^{2} \omega_{j}^{2} \\
\vdots \\
\sum_{j}^{N^{\prime \prime}} a_{n j} a_{i j} \omega_{j}^{2}
\end{array}\right\}-v_{i}\left\{e_{i}\right\}+\left\{\begin{array}{c}
\sum_{j}^{n^{\prime \prime}} a_{1 j} a_{i j} \omega_{j}{ }^{2}+\sum_{j}^{n^{\prime \prime}} a_{i j} a_{j 1} \omega_{1}{ }^{2} \\
\vdots \\
\sum_{j}^{n^{\prime \prime}} a_{i j} \omega_{j}{ }^{2}+\sum_{j}^{\prime} a_{i j} a_{j i} \omega_{i}{ }^{2} \\
\vdots \\
\sum_{j}^{n^{\prime \prime}} a_{n j} a_{i j} \omega_{j}{ }^{2}+\sum_{j}^{n^{\prime \prime}} a_{i j} a_{j n} \omega_{n}{ }^{2}
\end{array}\right\}
\end{aligned}
$$


From (31)

$$
\begin{aligned}
& -\omega_{i}^{2}[I]\left\{\varphi_{i}\right\}+[\bar{K}]\left\{\varphi_{i}\right\}=-\omega_{i}{ }^{2}\left\{\begin{array}{c}
\sum_{j}^{n^{\prime \prime}} a_{i j} a_{j 1} \\
\vdots \\
\sum_{j}^{n^{\prime}} a_{i j} a_{j i} \\
\vdots \\
\sum_{j}^{n^{\prime \prime}} a_{i j} a_{j n}
\end{array}\right\}-v_{i}\left\{e_{i}\right\}+\left\{\begin{array}{c}
\sum_{j}^{n^{\prime \prime}} a_{i j} a_{j 1} \omega_{1}{ }^{2} \\
\vdots \\
\sum_{j}^{n^{\prime \prime}} a_{i j} a_{j i} \omega_{i}{ }^{2} \\
\vdots \\
\sum_{j}^{n^{\prime \prime}} a_{i j} a_{j n} \omega_{n}{ }^{2}
\end{array}\right\} \\
& +\omega_{i}{ }^{2}\left\{\begin{array}{c}
\sum_{j=n+1}^{N} a_{1 j} a_{i j} \\
\vdots \\
\sum_{j=n+1}^{N} a_{i j}^{2} \\
\vdots \\
\sum_{j=n+1}^{N} a_{n j} a_{i j}
\end{array}\right\}-\left\{\begin{array}{c}
\sum_{j=n+1}^{N} a_{1 j} a_{i j} \omega_{j}^{2} \\
\vdots \\
\sum_{j=n+1}^{N} a_{i j}^{2} \omega_{j}^{2} \\
\vdots \\
\sum_{j=n+1}^{N} a_{n j} a_{i j} \omega_{j}^{2}
\end{array}\right\}
\end{aligned}
$$

From (32)

$$
\begin{aligned}
p_{i}= & \sum_{j=n+1}^{N} a_{i j}^{2}\left(\omega_{i}{ }^{2}-\omega_{j}{ }^{2}\right) \\
& \left\{\varphi_{i}\right\}=\left\{\begin{array}{c}
\sum_{j}^{n \prime} a_{i j} a_{j 1} \\
\vdots \\
0 \\
\vdots \\
\sum_{j}^{n} a_{i j} a_{j n}
\end{array}\right\}+\left\{\begin{array}{c}
\sum_{j=n+1}^{N} a_{1 j} a_{i j}\left(\frac{\omega_{i}{ }^{2}-\omega_{j}{ }^{2}}{\omega_{1}{ }^{2}-\omega_{i}{ }^{2}}\right) \\
\vdots \\
0 \\
\vdots \\
\sum_{j=n+1}^{N} a_{n j} a_{i j}\left(\frac{\omega_{i}{ }^{2}-\omega_{j}{ }^{2}}{\omega_{n}{ }^{2}-\omega_{i}{ }^{2}}\right)
\end{array}\right\} i=1,2, \cdots n
\end{aligned}
$$

On substituting (15), (16), (28) and (33) into (29)

$$
\begin{gathered}
\left\{x_{i}\right\}=\left[\phi_{1}+\epsilon \sum_{j}^{N^{\prime}} a_{1 j} \phi_{j}, \phi_{2}+\epsilon \sum_{j}^{N^{\prime}} a_{2 j} \phi_{j} \cdots \phi_{n}+\epsilon \sum_{j}^{N^{\prime}} a_{n j} \phi_{j}\right]\left\{\left\{e_{i}\right\}+\epsilon\left\{p_{i}\right\}\right. \\
+\epsilon^{2}\left\{\phi_{i}\right\}=\phi_{i}+\epsilon \sum_{j=n+1}^{N} a_{i j} \phi_{j}+\epsilon^{2}\left\{\sum_{j}^{n^{\prime \prime}} a_{i j} a_{j 1} \phi_{1}+\sum_{j}^{n^{\prime \prime}} a_{i j} a_{j 2} \phi_{2}+\cdots 0 \cdot \phi_{i}\right. \\
+\cdots \sum_{j}^{n^{\prime \prime}} a_{i j} a_{j n} \phi_{n}+\sum_{j=n+1}^{N} a_{1 j} a_{i j}\left(\frac{\omega_{i}{ }^{2}-\omega_{j}^{2}}{\omega_{1}{ }^{2}-\omega_{i}^{2}}\right) \phi_{1}+\sum_{j=n+1}^{N} a_{2 j} a_{i j}\left(\frac{\omega_{i}{ }^{2}-\omega_{j}{ }^{2}}{\omega_{2}{ }^{2}-\omega_{i}^{2}}\right) \phi_{2} \\
+\cdots 0 \cdot \phi_{i}+\cdots \sum_{j=n+1}^{N} a_{n j} a_{i j}\left(\frac{\omega_{i}{ }^{2}-\omega_{j}{ }^{2}}{\omega_{n}{ }^{2}-\omega_{i}{ }^{2}}\right) \phi_{n}-\sum_{j=1}^{N^{\prime}} a_{i 1} a_{1 j} \phi_{j} \\
\left.-\sum_{j=1}^{N^{\prime}} a_{i 2} a_{2 j} \phi_{j} \cdots 0 \cdot \phi_{i}+\cdots-\sum_{j=1}^{N^{\prime}} a_{i n} a_{n j} \phi_{i}\right\}+\epsilon^{3}\{
\end{gathered}
$$




$$
\begin{aligned}
=\phi_{i}+\epsilon & \sum_{j=n+1}^{N} a_{i j} \phi_{j}-\epsilon^{2}\left(\sum_{j=n+1}^{N} \sum_{\substack{l=1 \\
\neq i}}^{n} a_{i l} a_{l j} \phi_{j}\right. \\
& \left.-\sum_{j=n+1}^{N} \sum_{\substack{l=1 \\
\neq i}}^{n} a_{i j} a_{l j}\left(\frac{\omega_{i}{ }^{2}-\omega_{j}^{2}}{\omega_{l}{ }^{2}-\omega_{i}{ }^{2}}\right) \phi_{l}\right)+\epsilon^{3}\{\quad j=1,2, \cdots n
\end{aligned}
$$

From (34) it is easy to see the type of convergence Rubenstein's method possesses. After one iteration, the eigenvalue is correct to order $\epsilon^{2}$ and the approximation to the eigenvectors is improved, in that the vectors multiplied by $\epsilon$ in (34) are all higher order eigenvectors $(j>n)$ than the ones that have been obtained. If the original approximation is good, then Rubenstein's method should rapidly approach to within any desired approximation of the correct eigenvalue and eigenvector. Whether the method possesses advantages over conventional methods for use on a digital computer is not possible to say from the analysis, and is probably best determined experimentally on a computer.

\section{REFERENCES}

(1) Caughey, T. K., "Classical Normal Modes in Damped Linear Dynamic Systems", Journal of Applied Mechanics, Paper No. 59-A-62.

(2) Caughey, T. K., and O'Kelly, M. E. J., General Theory of Vibration of Damped Linear Dynamic Systems, Report Dynamics Laboratory, California Institute of Technology 1963.

Division of Engineering and Appited Science

California Institute of Technology

Pasadena, California

Manuseript received July 10, 1964. 